\title{
THE IMPACT OF LARGE DATABASES ON THE KNOWLEDGE OF LATE-TYPE CLOSE BINARIES
}

\author{
C. Maceroni ${ }^{1}$
}

RESUMEN

Presentamos algunos resultados del análisis de las muestras de binarias eclipsantes que han aportado los sondeos de microlentes OGLE. Estos experimentos observaron millones de estrellas en la dirección del bulbo galáctico (OGLE-I) y de la Nube Menor de Magallanes (OGLE-II). Su completez ha permitido el descubrimiento de sistemas raros e interesantes. Ejemplo de ello es un nuevo grupo de binarias de largo período en la NMM con una gigante en contacto con el lóbulo crítico, la cual domina la variación de luz del sistema (" $\beta$-contacts"). Estos sistemas obedecen una relación período-luminosidad, y podrían ser utilizados como herramienta auxiliar e independiente para determinar distancias. Otro objeto interesante por sus implicaciones para el estudio de la pérdida de momento angular por frenado magnético, así como de la actividad estelar, es BW3 V38, el sistema de enanas $\mathrm{M}$ de más corto período conocido, que fue descubierto por el OGLE-I, y que será estudiado espectroscópicamente. Se discuten brevemente las perspectivas para la investigación de binarias cerradas que abren las misiones espaciales futuras, como COROT y Eddington.

\section{ABSTRACT}

This paper presents some results of the analysis of the eclipsing binaries samples that came out as by-products of the OGLE microlensing surveys. These experiments monitored millions of stars in the direction of the galactic bulge (OGLE-I), and of the Small Magellanic Cloud (OGLE-II). Their completeness allowed the discovery of rare and interesting systems. An example is a new group of long period binaries in the SMC with presumably a giant component in contact with the critical lobe, which dominates the systemic light variation (" $\beta$-contacts"). These systems obey a period-luminosity-color relation and could be used as an auxiliary, but independent, tool for distance determination. Another very interesting object, for its implications in the studies of angular momentum loss processes by magnetic braking and of stellar activity, is the system of shortest known period with M dwarf components, discovered by OGLE-I, BW3 V38, that is the target of a spectroscopic follow-up. The perspectives for close binary star research in view of future space missions, such as COROT and Eddington are briefly discussed.

\section{Key Words: BINARIES: CLOSE - BINARIES: ECLIPSING - ASTRONOMICAL DATA BASES: MIS- CELLANEOUS - SURVEYS}

\section{THE BY-PRODUCT CATALOGS OF VARIABLE STARS FROM MICROLENSING SURVEYS}

Some of the most fruitful developments of stellar research in the last decade are related to the microlensing surveys. Several independent experiments were started in the nineties, after the suggestion by Paczynski (1986) that "monitoring the brightness of a few million stars in the Magellanic Clouds over a time scale between two hours and two years could lead to a discovery of 'dark halo' objects between $10^{-6}$ and $10^{-2}$ solar mass". $\mathrm{A}(\mathrm{n}$ incomplete) list of the microlensing surveys should at least mention the Optical Gravitational Lensing Experiment (OGLE), the MAssive Compact Halo Object search (MACHO), and their French precursor EROS

\footnotetext{
${ }^{1}$ Osservatorio Astronomico di Roma, INAF, Italy.
}

(Experience pour la Recherche d'Objets Sombres).

Obviously the monitoring of large stellar samples has provided an enormous amount of by-product data on stellar variability, which have the advantages of long-baseline stable photometry, of homogeneity and of completeness control. The best organized and easily accessible data are those from the OGLE surveys, all available over the Internet at http://sirius.astrouw.edu.pl/ ogle/, where links to the other experiments can also be found. The results presented in this paper are all based on the OGLE by-product catalogs.

The OGLE experiment is currently in its third phase (OGLE-III). The first one, OGLE-I (Udalski et al. 1992, for the technical details) observed in a pencil beam search volume towards Baade's window. The survey covered sixteen $15^{\prime} \times 15^{\prime}$ fields, 


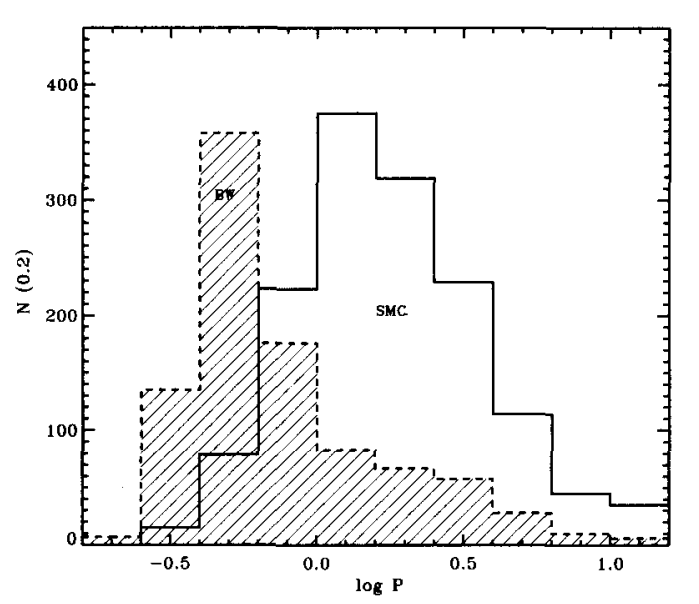

Fig. 1. The orbital period distribution of the Baade's Window sample (shaded histogram) and of the SMC sample as derived from OGLE-I and OGLE-II catalogs.

and provided $I$ band photometry of objects with $14 \leq m_{I} \leq 18$, and accuracy of $0.01-0.02$ mag (at best). The periodicity search spanned the interval $0^{d} .01 \leq P \leq 100^{d}$. The by-product data on variable stars were released in the form of a catalog (Udalski et al. 1997a, and references therein) that lists $\sim 1300$ eclipsing binaries. For each entry the position, the orbital period, the $I$ light curve and the $V-I$ color at maximum are available. The completeness of the catalog, as estimated from independent detection of the same systems in overlapping fields, depends of course on the object magnitude and has a mean value of $88 \%$.

During the second phase OGLE II, an essentially similar experiment with higher performance and dedicated instruments, monitored fields in the Magellanic Clouds and the bulge (Udalski et al. 1997b). A catalog of variables similar to that of OGLE-I and containing 1500 eclipsing systems in the Small Magellanic Cloud (SMC) was published by Udalski et al.(1998).

Figure 1 shows the orbital period distribution for the eclipsing binaries of the first 10 fields of the OGLE-I catalog (BWC + BW1-BW9) and those of the SMC catalog. The lack in the latter of short period binaries is certainly a distance effect, as shorter period systems belonging to the SMC contain components fainter than the OGLE-II magnitude limit.

\subsection{The eclipsing binaries in the Baade's Window}

The sample of 1300 eclipsing binaries of OGLE-I contains a majority (72\%) of contact binaries whose properties were studied in great detail by Rucinski (1997a,b; 1998a,b). Among the main results we can mention: the fact that $\mathrm{W}$ UMa contact binaries can

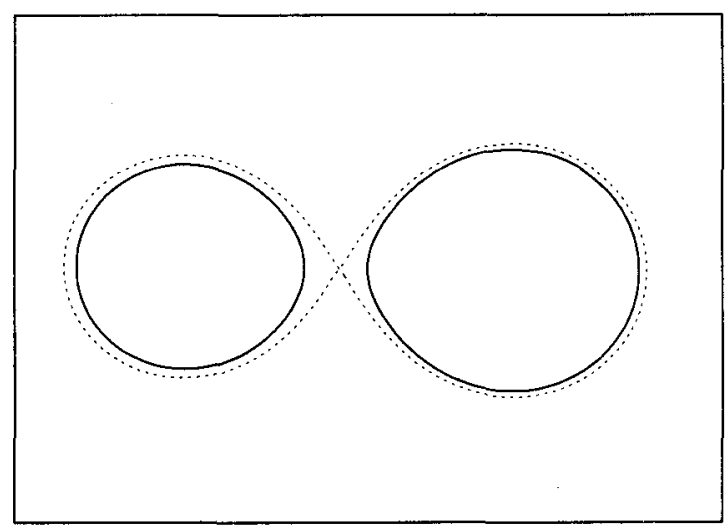

Fig. 2. The configuration (projection on the orbital plane) of the BW3 V38 model from MR97, the primary fills $86 \%$ and the secondary $76 \%$ of the respective Roche lobe volume.

be used as distance tracers all the way to the galactic bulge, their belonging to the old galactic disc population and the updating of the apparent frequency of contact binaries.with respect to MS stars, with a peak at short periods with respect to the extrapolation of the Duquennoy and Mayor (1991) distribution. This feature has a straightforward explanation in terms of a relatively long lived contact stage, related to a possible decrease of magnetic braking mechanisms after contact. A related investigation, on the period distribution of pre-contact

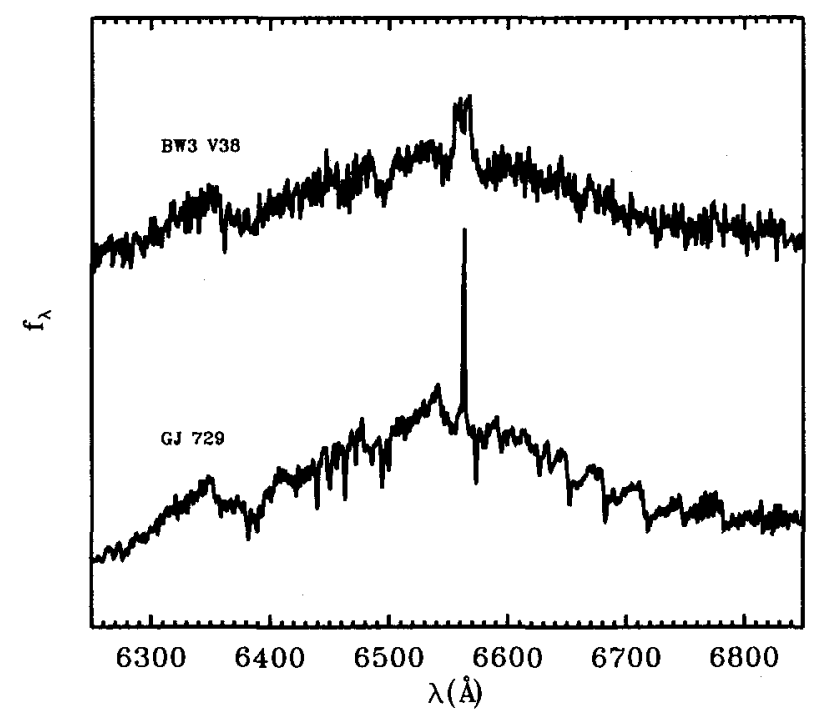

Fig. 3. Upper curve: the NTT/EMMI spectrum of BW3 V38 around quadrature $(\varphi=0.272)$ compared to the template spectrum of the M dwarf GJ 729 (M3.5 V). The splitting of $\mathrm{H} \alpha$ is clearly visible and indicates emission from both components. 

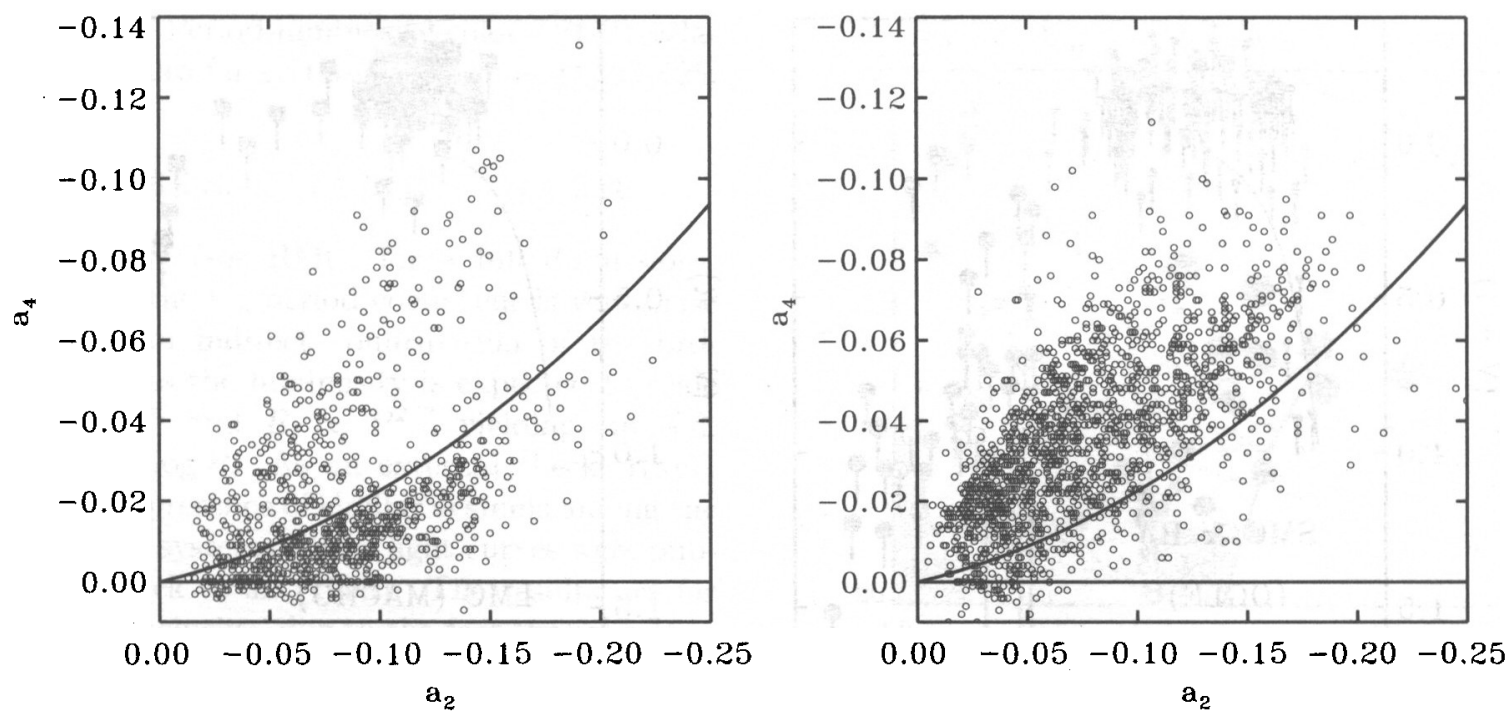

Fig. 4. The location of the OGLE-I and OGLE-II eclipsing systems in the $a_{2}-a_{4}$ Fourier coefficients diagram. Contact systems are found below the continuous line (contact locus), as defined by Rucinski (1993).

late-type systems in the OGLE-I catalog was carried out by Maceroni and Rucinski (1999). This was in part an update of a previous study by Van't Veer and Maceroni (1988) and Maceroni and Van't Veer (1991), by means of the more homogeneous and complete OGLE data. The logarithmic slope of the period distribution was used to infer the angular momentum loss efficiency (and consequently the functional form of the magnetic braking law) for late, rapidly rotating members of tidally locked binaries. These results also show a change in the braking mechanisms ("saturation") for fast rotating components approaching the contact stage, in agreement with the most recent studies on stellar activity (Pizzolato et al. 2003).

An interesting case study was dedicated by Maceroni and Rucinski (1997, hereafter MR97) to the system BW3 V38 (i.e the variable \# 38 of the third Baade's Window field). This is the eclipsing system of shortest known period $\left(0^{d} .1984\right)$ with a light curve with minima of almost equal depth, $\Delta I \simeq 0.75$, and very red color, $(V-I)=2.45$. These features suggest that the binary might be formed by two similar red dwarfs (the dereddened color from MR97, $(V-I)_{0}=2.3$, corresponds to a M3-M4 spectral type). The light curve solution by MR97 yielded indeed a configuration with similar components (mass ratio $q=m_{1} / m_{2}=0.77$ and effective temperatures, respectively, $\mathrm{T}_{1}=3500 \mathrm{~K}$ and $\mathrm{T}_{2}=3459 \mathrm{~K}$ ). The stars turned out to be very close but not yet in contact with the Roche critical surface, as shown in Figure 2. Therefore, this appears to be the first system with dwarf $M$ type components caught while approaching contact; a better knowledge of its physical properties will shed light on the poorly known subject of angular momentum loss in $M$ type dwarfs. Even if the limited accuracy of the OGLE photometry and of the light curve solution does not completely rule out a contact configuration, a further study is anyway of great interest. It is well known that the period distribution of the $\mathrm{W}$ UMa contact binaries shows a sharp cut-off at $P=0^{d} .23$ with CC Com (spectral type K5), a fact that has been the object of several investigations but is not yet completely explained. If BW3 V38 turns out to be a contact binary, then it will be the first case of a system beyond the period cut-off. For these reasons a program of mid-resolution spectroscopic observations for spectral classification and radial velocity study is on the way, Maceroni (2003, in preparation). A series of 34 mid-resolution spectra were acquired at the ESO New Technology Telescope (NTT) with EMMI in spectroscopic mode. The preliminary results confirm the spectral type as derived by the color information, see Figure 3, where a spectrum of the binary at quadrature is compared with that of a M3.5 V template star. The splitting of $\mathrm{H} \alpha$ in emission confirms the similarity (also concerning surface activity) between the two components.

\subsection{The $\beta$-contact binaries in the $S M C$}

As suggested by Figure 1 the OGLE-II sample of eclipsing binaries should contain intrinsically brighter stars than that of BW. This is confirmed by the study of the sub-sample of contact binaries, 

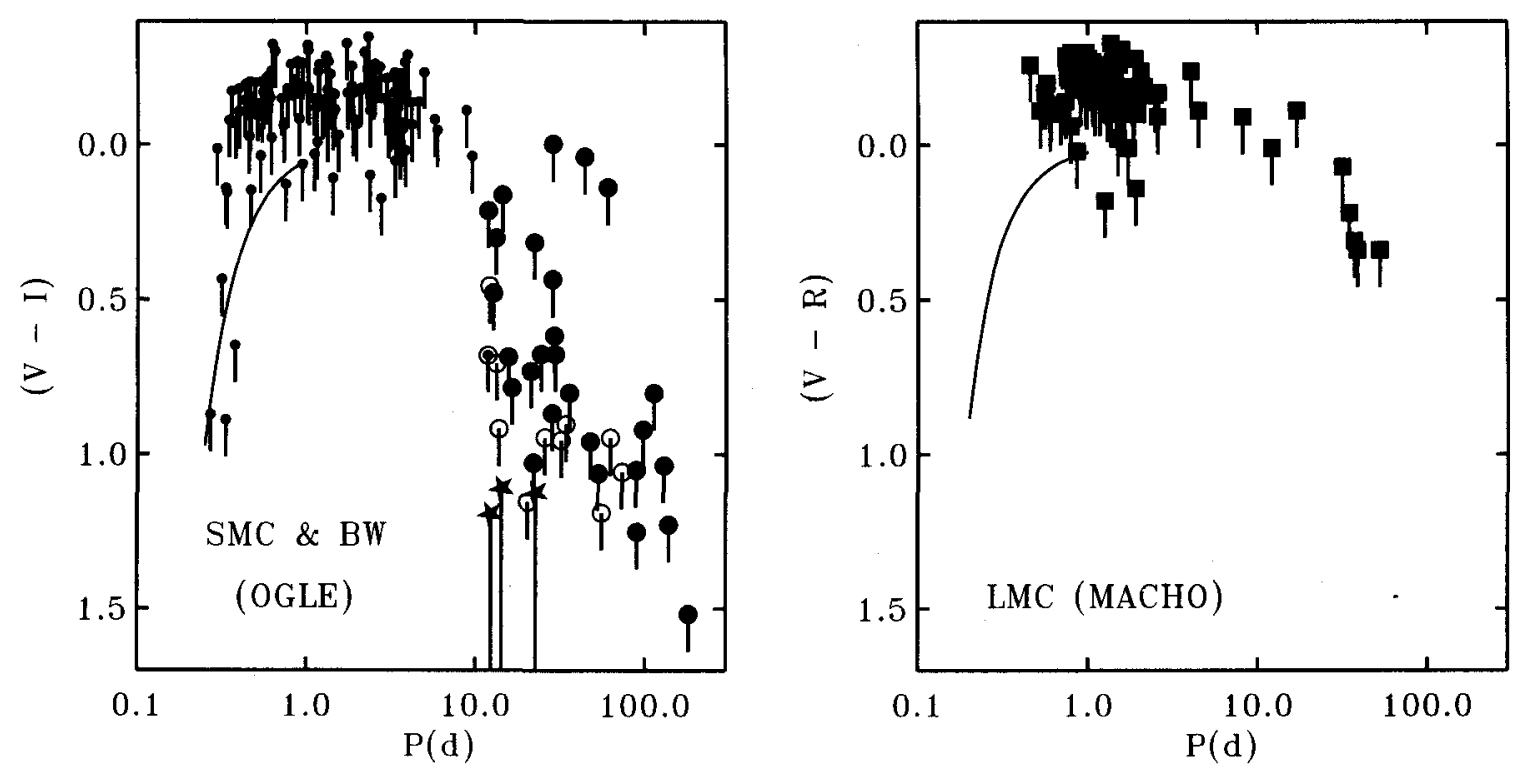

Fig. 5. The period-color diagram for all contact systems in the SMC (left panel) and LMC (right panel) from RM01. The open circles mark systems that were assigned half weights in the luminosity calibrations. The large stars in the left panel mark the three long period contact binaries of the OGLE-I sample. The SMC data are based on the $(V-I)$ color index while the LMC data are based on the $(V-R)$ color index. The vertical segments indicate the displacement due to reddening correction. For simplicity, the same reddening vectors were assumed for all systems in the $\mathrm{SMC}, E_{V-I}=0.12$. The curves give the blue short-period envelope for normal, short-period W UMa-type systems.

that is numerically much less conspicuous than the BW sample. The latter turned out to outnumber all other binary types and to be essentially composed of W UMa binaries (contact systems with late-type unevolved components).

The selection of contact systems, just on the basis of the light curve shape, can be efficiently performed by means of an automatic filtering algorithm that was originally introduced by Rucinski (1993). A "contact locus" can be defined in a $a_{2}-a_{4}$ diagram, where the $a_{i}$ 's are the second and fourth cosine coefficients of the Fourier decomposition of the light curve according to: $\ell(\varphi)=\sum_{i=0}^{\infty} a_{i} \cos (2 \pi i \varphi)$. This "locus" separates the area where models of contact binaries are found-see Figure 6 in Rucinski's (1993) paper-and it only weakly depends on the atmospheric properties of the stars, as the light variation in a contact configuration is due mainly to the effects of the distortion of the stellar surfaces. Figure 4 shows the $a_{2}-a_{4}$ diagram for all systems of the OGLE-I and the OGLE-II catalogs. The contact systems are found in the region below the curve. Figure 4 confirms that the observable SMC sample is mainly formed by non-contact (and bright) systems. A more intrinsic difference between the two samples turns out when the Period - Color (P-C) diagram is considered for the systems selected as contact binaries (Rucinski and Maceroni 2001, hereafter RM01).
The contact binaries of OGLE-I are relatively short period systems as only three objects have $P>10^{d}$. Besides, they follow the typical P-C relation, discovered by Eggen (1961) for field contact binaries, by which larger periods correspond to bluer color. That is believed to express the scaling of the system properties with the mass (and size) of its unevolved components. Mochnacki (1981) pointed out that a short-period-blue-envelope locus can be defined, corresponding to the minimum contact period for ZAMS components.

The SMC sub-sample coming out of the Fourier filter, is formed by a "normal" group of short period contact binaries, but as well by a group with $10^{d} \leq P \leq 200^{d}$ showing an "inverted" $\mathrm{P}-\mathrm{C}$ relation, in the sense that longer periods correspond to redder colors. That property, together with the typical shallow light curve of those systems (see RM01, Figure 2) suggests that the system light variations are essentially of ellipsoidal origin, and due to a giant component in contact with the lobe. Semi-detached systems of this type do indeed pass the Fourier filter, as long as the star in contact dominates the system light. The position of these " $\beta$-contact" systems (from the $\beta$ Lyr-type light curves) in the CM diagram (Figure 6) is in agreement with this hypothesis.

Another interesting property of this sample is the 
existence of a Period-luminosity-color (PLC) relation, that applies for systems with $(V-I)_{0}>0.25$, in the form:

$$
M_{V}=-3.43 \log P+2.04(V-I)_{0}+2.80
$$

with $\sigma=0.34$ (see RM01 for a full discussion). The fact that the log-period coefficient is very close to $-10 / 3$ is an indirect confirmation of the proposed model, as the luminosity is expected to scale as $L \propto R^{2} T^{4}$ and $R \propto P^{2 / 3}$ yielding, indeed, $M_{V} \propto-10 / 3 \log P$. As an external check, RM01 looked for the presence of similar systems among the LMC eclipsing systems whose light curves were published by Alcock et al. (1998). The results are far from being conclusive, due to the fact that the MACHO sample is strongly biased towards large amplitude systems, as Alcock et al. (1998) excluded from their analysis the variables with light curve amplitude $M_{R}<0.2$. Moreover, the total number of systems is also smaller by a factor 2.5 and, therefore, only a few (7) systems are found with $P>10^{d}$; those provide however some support to the hypothesis (see Figure 4, right panel).

The $\beta$-PCL relation (from $\beta$-contacts) can be used as an auxiliary - but independent - tool for distance determination to nearby galaxies. Though these binaries are much less numerous than the classical distance indicators ( $1 / 50$ with respect to Cepheids from the numbers in OGLE-II), they are very bright objects, easy to detect (because of the continuous variability) and yield a completely independent check.

\section{THE FUTURE DATABASES FROM SPACE EXPERIMENTS}

A further advance in the richness and quality of the databases of variable stars will mark the current decade, when the data of new space missions will be released. The first ones will be those of COROT and Eddington satellites, which will be devoted to asteroseismology and exo-planet searches, but as well to parallel science programs, where the studies of variability, and of eclipsing binaries in particular, fit perfectly.

\subsection{COROT}

The acronym COROT ${ }^{2}$ stands for COnvection ROtation and Transits. It refers to a French-led European "small" space mission, whose goals will be achieved by means of ultra-high precision (100 ppm), wide field, relative photometry obtained with

\footnotetext{
${ }^{2}$ more details can be found on the official COROT website at ww.astrsp-mrs.fr/projets/corot/
}

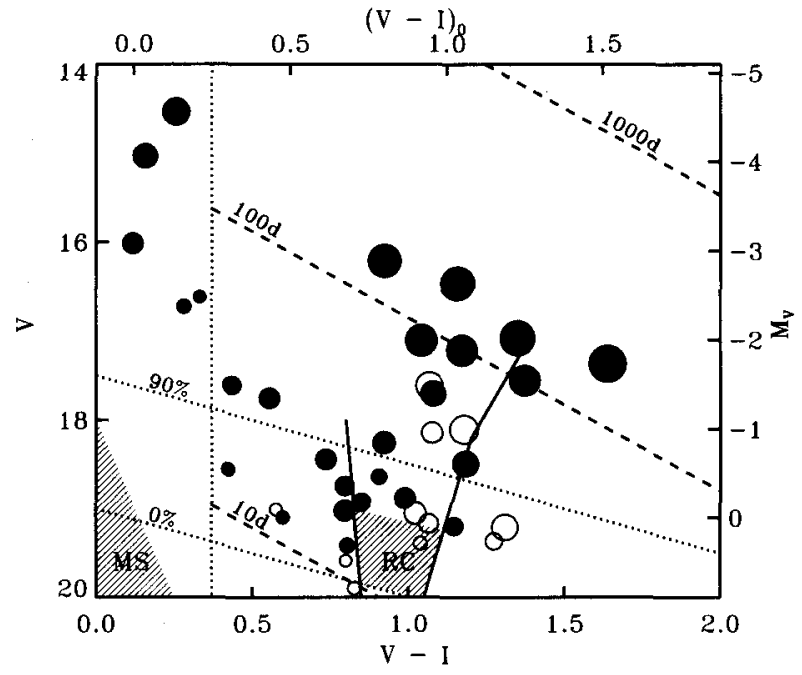

Fig. 6. The color-magnitude diagram for the $\beta$-contact systems in the SMC. The orbital periods of individual systems are roughly proportional to the circle size. The right and upper axes show the de-reddened color index and absolute magnitude data for $E_{V-I}=0.12$ and $(m-M)_{0}=18.8$. The slanted dotted lines give the approximate locations of the $90 \%$ and $0 \%$ completeness levels of the OGLE-II variable star search. The broken lines represent the constant period lines according to the calibration of the PLC- $\beta$ relation as in RM01.

long continuous observing runs on the same field. The COROT instrument is a white-light wide-field photometer with an entrance pupil of $27 \mathrm{~cm}$ and a set of four frame-transfer CCD detectors (for a total field of $\left.2.6^{\circ} \times 3^{\circ}\right)$. Two CCDs are devoted to asteroseismology (and collect defocussed, integral light), and two to exoplanet search (focussed light and color information-thanks to a mini-prism-for $V<15$ objects). COROT will be launched at the end of 2005 on a low-earth polar inertial orbit, allowing it to monitor stellar fields near the pole of the orbit continuously for about 5 months. The mission lifetime will be nominally 3 years, corresponding to 5-6 "long runs" $\left(150^{d}\right)$. Shorter $10-20^{d}$ runs are foreseen between two consecutive long ones. While the seismology field will be entirely devoted to the observation of very few pre-selected targets, the exo-planet observations will consist of monitoring the variability of a large number of stars (12000 for field and run) with a time sampling of $8^{m}$ or, in selected cases, up to $32^{s}$. The final outcome will be therefore a large number $(\sim 70,000)$ of high precision - long time-span light curves.

\subsection{Eddington}

Eddington is an ESA mission scheduled for early 2008. Its goals are similar to, but more ambitious 
than, those of COROT, thanks to the higher-class instrumentation (and larger light-collecting area). According to the current baseline, its payload ${ }^{3}$ will consist of "a white-light, broad band, wide-field photometer, implemented as a set of 4 identical, coaligned wide field optical telescopes with a mosaic CCD camera at its focal plane". The telescopes will have a diameter of $60 \mathrm{~cm}$ and will look at the same stellar field. The diameter of the total field of view will be $6.72^{\circ}$.

The main advantages with respect to COROT will be:

- a larger light collecting area, implying a signalto-noise ratio improvement $S / N>3$,

- a larger ( 2 times) field of view,

- a shorter time sampling ( $30^{s}$ versus COROT's $8^{m}$ for most objects)

- a different mode of observation: COROT will simultaneously acquire data for both core programs, with the exoplanetary field strongly constrained by the choice of the seismology program primary targets. Eddington will adopt instead a sequential mode; the planet search will be performed in the second part of the mission, monitoring, for three continuous years, an expressly selected field.

At the end of the mission accurate photometry of $\sim 200,000$ stars of any spectral type and luminosity class is expected.

\subsection{The potentials for eclipsing binary research}

The numerous possible advances related to the next asteroseismological missions have already been reviewed during several workshops dedicated to the various experiments. (A rich section on binaries is found, for instance, in the proceedings of the $3^{\text {rd }}$ MONS Workshop ${ }^{4}$, a small Danish mission that was unfortunately canceled).

In short, the most rewarding results will directly descend from the two main features of these space experiments: high precision photometry and long continuous monitoring.

The high precision photometry will allow a better determination of the basic stellar parameters (masses, radii) that will be obtained from observations of well-behaved systems, i.e. preferentially detached binariesData are still needed for lower MS, pre-MS and giant stars. In particular the lower MS calibration was based until recent years on only two

\footnotetext{
${ }^{3}$ the full description is available at: http://astro.esa.int/SA-general/Projects/Eddington/baseline.html

${ }^{4}$ available at http://astro.ifa.au.dk/ $\sim$ tct/MONSworkshop3/Proceedings/List_Contents.html
}

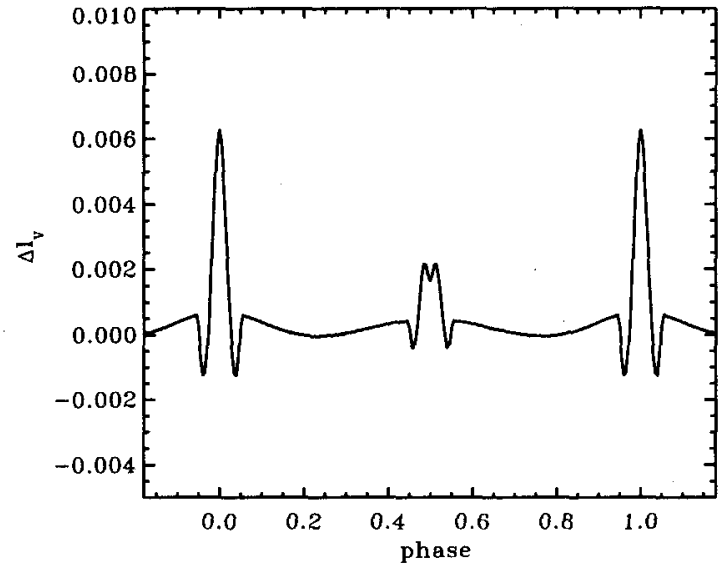

Fig. 7. The difference between two synthetic light curves obtained by changing the linear (V) limb darkening coefficient from 0.57 to 0.67 . The corresponding system is a model of V805 Aql, according to Popper (1981), i.e. a detached binary with relatively short period and MS components $\left(P=2^{d} .41\right.$, effective temperatures $\left.T_{p}=8164 \mathrm{~K}, T_{s}=7178 \mathrm{~K}\right)$. The other parameters are: mass ratio $q=m_{s} / m_{p}=0.81$, inclination $i=86^{\circ}$, fractional radii $r_{p}=0.18, r_{s}=0.15$, fractional luminosity $L_{s} / L_{p}=0.362$. The synthetic light curves were computed with the 1995 version of the Wilson and Devinney code.

eclipsing binaries, CM Dra and YY Gem. Recent interferometric results (Lane, Boden \& Kulkarni 2001; Ségransan et al. 2003) have provided accurate radii for several $\mathrm{M}$ dwarfs. However the classical method based on spectroscopic eclipsing binaries, still has the advantage of a simultaneous determination of mass and radius. Eddington will also observe several open clusters (Pleiades, Hyades, $\alpha$ Per), with all the advantages deriving from chemically homogeneous and coeval samples.

Besides, the analysis of the accurate light curves will allow a direct determination of stellar atmosphere properties, such as limb darkening and, for tidally distorted binaries, gravity darkening. These are second order effects in the light curves, that are thus far usually assumed in light curve solutions, mainly because of insufficient data accuracy. Figure 7 shows, just as an example, the effect on the synthetic light curve of a change of 0.1 in the (linear) limb darkening coefficient. The corresponding binary model has close but detached MS components, i.e., with light curves showing small "proximity effects" $\left(P=2^{d} .41\right)$. The difference between the curves is at least an order of magnitude larger than COROT's expected accuracy. It has to be taken into account, however, that only the light curve phases corresponding to the eclipses are sensitive to the limb 
darkening value; in the given example the eclipse duration is $0.1 \mathrm{P}$, i.e. $\sim 6$ hours. The typical COROT time-sampling of $8^{m}$ would still provide a good time coverage in this case, but a faster one would be necessary for shorter durations.

The long continuous monitoring will allow the study with higher accuracy data of the manifestations of stellar activity (spots, flares, stellar activity cycles) and indirectly of rotational period and differential rotation (from spot migration) in late -type componfistis).

Asteroseismology studies in binaries (mainly with Eddington since so far no binary is among the asteroseismological targets selected for COROT) would also be possible (see, for instance, Mkrtichian et al. 2003). In principle, looking for solar-type oscillation in suitable eclipsing binaries would have the advantage of knowing the masses, radii and the orbit inclination angle, and therefore the rotational velocity.

Finally the large photometric databases will allow archival programs, dedicated to the study of the by-product binary samples and of individual new interesting systems. Anyway, to exploit the opportunities provided by the missions fully, parallel (or follow-up) programs of target characterization and of high dispersion and high $\mathrm{S} / \mathrm{N}$ spectroscopy should urgently be planned. So far, only the programs on binaries related to surface activity studies benefit from a ground-based observational program for target characterization (Pagano et al. 2001). The further development of automatic selection algorithms will also be extremely useful, in view of the amount of data that will be provided by the missions.

I am grateful to the Scientific Organizing Committee and the International Astronomical Union for the allocation of a generous grant that made possible my participation in the Colloquium. I wish to thank the OGLE team for access to their database, Slavek Rucinski for his continuous encouragement, and Annie Baglin, Werner W. Weiss and Fabio Favata for providing updated information on the COROT and Eddington experiments.

\section{REFERENCES}

Alcock, C. et al. 1998, AJ, 115, 1921

Duquennoy, A., \& Mayor, M. 1991, A\&A, 248, 485

Eggen, O. J. 1961, London, H. M. Stationery Off., 1961.,

Lane, B. F., Boden, A. F., \& Kulkarni, S. R. 2001, ApJ, $551, \mathrm{~L} 81$

Maceroni, C., \& Rucinski, S. M. 1997, PASP, 109, 782 (MR97)

Maceroni, C., \& Rucinski, S. M. 1999, AJ, 118, 1819

Maceroni, C., \& Van't Veer, F. 1991, A\&A, 246, 91

Mkrtichian, D. E., Nazarenko, V., Gamarova, A. Yu., Lehmann, H., Rodriguez, E., Olson, E. C., Kim, S.-L., Kusakin, A. V., \& Rovithis-Livaniou, H. 2003, ASP Conf. Ser. , 292 "Interplay of Periodic, Cyclic and Stochastic Variability in Selected Areas of the H-R Diagram", ed. Sterken, C. (San Francisco: Astronomical Society of the Pacific), 113

Mochnacki, S. W. 1981, ApJ, 245, 650

Paczynski, B. 1986, ApJ, 304, 1

Pagano, I. et al. 2001, available at http://www.astrsp-mrs.fr/projets/corot/meeting/rodono_activ.pdf

Pizzolato, N., Maggio, A., Micela, G., Sciortino, S., \& Ventura, P. 2003, A\&A, 397, 147

Popper, D. M. 1981, ApJ, 244, 541

Rucinski, S. M. 1993, PASP, 105, 1433

Rucinski, S. M. 1997a, AJ, 113, 407

Rucinski, S. M. 1997b, AJ, 113, 1112

Rucinski, S. M. 1998a, AJ, 115, 1135

Rucinski, S. M. 1998b, AJ, 116, 2998

Rucinski, S. M. \& Maceroni, C. 2001, AJ, 121, 254 (RM01)

Ségransan, D., Kervella, P., Forveille, T., \& Queloz, D. 2003, A\&A, 397, L5

Udalski, A., Szymanski, M., Kaluzny, J., Kubiak, M., \& Mateo, M. 1992, Acta Astronomica, 42, 253

Udalski, A., Olech, A., Szymanski, M., Kaluzny, J., Kubiak, M., Mateo, M., Krzeminski, W., \& Stanek, K. Z. 1997a, Acta Astronomica, 47, 1

Udalski, A., Kubiak, M., \& Szymanski, M. 1997b, Acta Astronomica, 47, 319

Udalski, A., Soszynski, I., Szymanski, M., Kubiak, M., Pietrzynski, G., Wozniak, P., \& Zebrun, K. 1998, Acta Astronomica, 48, 563

Van't Veer, F. \& Maceroni, C. 1988, A\&A, 199, 183

C. Maceroni: Osservatorio Astronomico di Roma, Istituto Nazionale di Astrofisica, Monteporzio C. (RM)), I-00040, Italy (maceroni@mporzio.astro.it). 


\section{DISCUSSION}

Lampens - What kind of specific tools do you need for the treatment of the binary data in the Eddington project?

Maceroni - We need algorithms for classification of different binary types, for instance. Also, updated codes for light curve analysis, including updated model atmospheres.

Richichi - In the last two years a number of K-M dwarf stars have been measured by interferometry, providing additional calibrations for stellar radii on the late main sequence. Can you comment?

Maceroni - That decreases, of course, the importance of BW3V38 as a MS calibrator. However, it remains a very important object for checking the theories of evolution towards contact of late-type close systems.

Hummel - You mentioned the measurement of the linear limb-darkening coefficients. Will you be able to measure the precise limb-darkening profile also?

Maceroni - The example here is only with linear limb darkening. However, the light curve synthesis models offer other options (e.g., a logarithmic law). I suppose that with the accuracy of future data it will be possible to derive information on the profile as well.

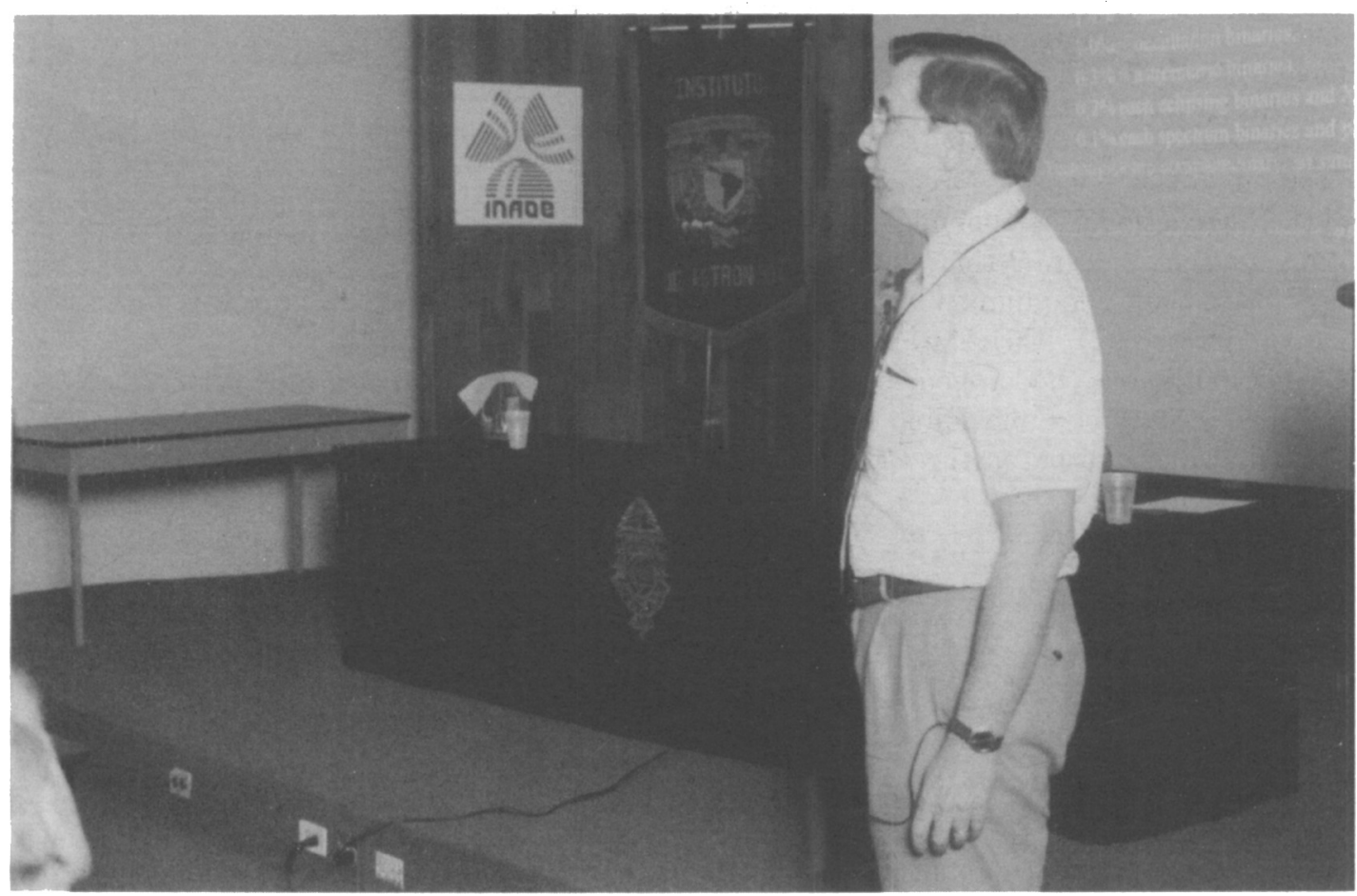

Bill Hartkopf. 